\title{
First Census of Galactic Molecular Clouds
}

\author{
Alisher S. Hojaev, Anna A. Kovaleva and Nigina R. Alimova \\ Dept. of Astronomy \& Astrophysics, National University of Uzbekistan, \\ Tashkent, 700174, Uzbekistan, email: hojaev@yahoo.com
}

\begin{abstract}
The first attempt at a census of all molecular clouds (MoC) in the Milky Way observed to date is presented. The catalog of MoC includes all observed and estimated physical parameters. The data will be used to compute a model of the MoC system in the Galaxy.
\end{abstract}

Keywords. Galaxy: structure — ISM: clouds — ISM: molecules

Nowadays the quick progress in instrumentation and observing technology provides possibilities to find and deeply study the interstellar medium (ISM) not only in our Galaxy but also in the nearest galaxies such as the Andromeda galaxy, the Magellanic Clouds and others. For example recent millimeter wave observations of M31 using the BIMA interferometer (Rosolowsky 2007) have revealed 67 giant MoC, extending along the spiral arms of the Andromeda nebula. $\mathrm{MoC}$ are investigated in detail in the relatively close galaxies, while the giant $\mathrm{MoC}$ might be studied in relatively distant extragalactic objects as well (for example, in spiral galaxies such as M33, M63, M64; in dwarf irregular galaxies such as IC 10, NGC 6822, NGC 1569, etc.). The results of observations of GMCs in the nearest galaxies with the Nobeyama 45 meter radio telescope in preparation for ALMA projects were recently published by Kuno et al. (2008).

Molecular clouds and the closely related cold dust-gas clouds play a key-role in the star forming processes as well as in the kinematics and dynamics of the Galaxy as a whole. These arguments show the importance of counting and surveying the MoC populations. In order to attempt to solve at least some problems of the physics and evolution of the MoC system in our Galaxy (as well as in other galaxies), its impact on the dynamics and evolution of the Galaxy itself, and to extend the results to $\mathrm{MoC}$ systems in other galaxies, we started to draft a consolidated catalog of molecular and dust-gas clouds that are observable from the Earth based on the recent data.

Online data banks and services such as VizieR, SIMBAD at CDS as well as original papers, reports and other publications were used. In our Galaxy there are about 200 large molecular clouds and more than 2500 smaller cold dark clouds (including clumps and cores this value exceeds approximately 5000 objects) observed in the Solar vicinity and neighborhood up to $11 \mathrm{kpc}$. The general catalog has been divided into 3 sub-catalogs: 1) large and giant $\mathrm{MoC} ; 2$ ) MoC with moderate masses and sizes; 3) small MoC including the clumps and cores. All main catalogs and subcatalogs contain the coordinates, sizes, distances, masses and other physical parameters (such as density, temperature, radial velocity, etc.) that are available for the different clouds. A preliminary statistical analysis of the data has been performed, the spatial distribution is drawn and the total number is estimated. We compare and discuss our results with data of other investigations as well as propose ways to complete and improve the catalog data.

\section{References}

Rosolowsky, E. 2007, ApJ, 654, 240

Kuno, N., Hirota, A., Tosaki, T., \& Miura, R. 2008, Ap\&SS, 313, 293 